DOI: $10.4274 /$ jarem.galenos.2020.3885

J Acad Res Med 2020;10(3):269-76

\title{
Follow-up for Patients with Intestinal Metaplasia Restricted to the Antrum
}

\author{
Deniz Öğütmen Koç11, (1) Sadettin Hülagü2, (1) Yeşim Saliha Gürbüz33, (1) Altay Çelebi2, (1) Ali Erkan Duman², \\ (1) Tanyeli Güneyligil Kazaz4 \\ ${ }^{1}$ University of Health Sciences Turkey, Gaziosmanpaşa Training and Research Hospital, Clinic of Gastroenterology, İstanbul, Turkey \\ ${ }^{2}$ Kocaeli University Faculty of Medicine, Department of Gastroenterology, İzmit, Turkey \\ ${ }^{3}$ istinye University Faculty of Medicine, Department of Pathology, İstanbul, Turkey \\ ${ }^{4}$ Gaziantep University Faculty of Medicine, Department of Biostatistics, Gaziantep, Turkey
}

Cite this article as: Öğütmen Koç D, Hülagü S, Gürbüz YS, Çelebi A, Duman AE, Güneyligil Kazaz T. Follow-up for Patients with Intestinal Metaplasia Restricted to the Antrum. J Acad Res Med 2020;10(3):269-76

\begin{abstract}
Objective: Guidelines recommend endoscopic surveillance for patients with extensive atrophy/intestinal metaplasia (IM), but follow-up is not recommended for patients with atrophy/IM restricted to the antrum. We evaluated the risk of neoplastic lesions in patients with antrum-restricted IM to determine whether surveillance endoscopy is necessary.

Methods: Overall, 117 patients with antrum-restricted IM diagnosed within the past 10 years underwent surveillance endoscopy. The gastric biopsy specimens were evaluated for atrophy, IM, and dysplasia.

Results: We enrolled 117 patients. Surveillance endoscopy was performed at a median (interquartile range) of 7.2 years (5.9-8.7 years) after the initial diagnosis of IM. On surveillance endoscopy, $27.4 \%$ of patients exhibited progression in their IM grade, whereas $25.6 \%$ had atrophy progression, and $33.3 \%$ had dysplasia progression. High-grade dysplasia and gastric cancer (GC) were detected in four and two patients, respectively. The annual incidence of GC in patients with antrum-restricted IM was $0.17 \%$. IM grade and type regressed in $29.9 \%$ and $38.5 \%$ of patients, respectively. Most patients with progressive IM grade, IM type, and dysplasia on surveillance endoscopy had Operative Link on Gastritis Assessment (OLGA) stage 3-4 $(p=0.0001, p=0.008$, and $p=0.0001$, respectively), and most patients with progressive atrophy and dysplasia had Operative Link on Gastric IM (OLGIM) stage 3-4 (both $p=0.001)$.

Conclusion: Patients with IM restricted to the antrum are at risk for neoplastic lesions and require endoscopic surveillance, contrary to existing recommendations. Premalignant lesions can exhibit both progression and regression. Therefore, a patient-specific surveillance program based on OLGA and OLGIM might be appropriate.
\end{abstract}

Keywords: Gastric cancer, intestinal metaplasia, surveillance endoscopy

\section{INTRODUCTION}

Gastric cancer (GC) is one of the most common cancers globally and has a poor prognosis, especially in the advanced stages of the disease. Nevertheless, screening and monitoring patients at risk of GC can facilitate early detection and treatment and reduce mortality (1).

Intestinal-type gastric adenocarcinoma is the final stage of a multistage disease process known as the Correa cascade, which includes

ORCID IDs of the authors: D.Ö.K. 0000-0003-2738-625X; S.H. 0000-0001-6659-1286; Y.S.G. 0000-0002-4278-8241; A.Ç. 0000-0003-2864-7124;

A.E.D. 0000-0002-7093-2503; T.G.K. 0000-0002-4191-1244. 
inflammation, atrophy, intestinal metaplasia (IM), dysplasia, and carcinoma (2). IM is a precancerous lesion characterized by replacement of the epithelium in the oxyntic or antral mucosa with intestinal epithelium. IM is classified as complete (small-intestine type) or incomplete (colonic type; thought to be the most advanced stage of IM) based on the histologic characteristics and type of mucinous material secreted (3). Notably, patients with gastric IM, along with other precancerous gastric lesions, are better monitored in Asia than in Europe (4). New guidelines for screening programs were published recently in Western countries $(5,6)$. However, a study conducted in the United States revealed that $78 \%$ of endoscopists were not aware of the guidelines for the surveillance and management of IM (7). Notably, the best strategy for reducing mortality in patients at high risk of $\mathrm{GC}$ is diagnosis and surveillance of precancerous gastric lesions $(8,9)$. The recently presented guidelines recommend endoscopic surveillance for patients with extensive atrophy and/or $\operatorname{IM}(5,6,10)$. However, no scheduled endoscopic and histologic surveillance was recommended for patients with antrum-restricted IM and atrophy $(6,11)$. However, to the best of our knowledge, no comprehensive study has been conducted regarding the follow-up of patients with premalignant gastric lesions restricted to the antrum.

Our study evaluated the risk of neoplastic lesions in patients with antrum-restricted IM to determine whether surveillance endoscopy is necessary.

\section{METHODS}

For this single-center study, we invited patients with antrumrestricted IM that had been histologically confirmed with untargeted biopsy sampling in the past 10 years to undergo targeted biopsy sampling during surveillance endoscopy. We selected patients with at least 4 years between the initial and surveillance endoscopies. Overall, 607 patients were identified. We excluded patients with peptic ulcers, Barrett's esophagus, GC, other cancers, and prior gastric resection. In addition, we excluded patients whose initial gastric biopsy did not meet the minimum quality criteria (e.g., paraffin block for a reassessment of the antrum mucosa) and those who could not be reached by telephone. We called up 182 patients and invited them to undergo surveillance endoscopy; of these, 117 agreed and were included in the study. During the appointment, patients were asked about their smoking history, use of non-steroidal anti-inflammatory drugs (NSAIDs), use of proton pump inhibitors (PPIs), diagnosis and treatment of Helicobacter pylori (H. pylori) infection, and family history of GC. For consistency, all esophagogastroduodenoscopy and biopsy procedures were performed by a single physician (D.O.K.).

For optimal assessment of the severity and distribution of premalignant gastric lesions, biopsies were obtained from five standardized intragastric locations during surveillance endoscopy according to a predetermined protocol (12) (Figure 1). Overall, the following 12 biopsies were obtained: 4 from the pylorus $2-3 \mathrm{~cm}$ proximal to the antrum, 2 from the opposite walls of the incisura angularis, 2 from the corpus minor curvature, 2 from the corpus greater curvature, and 2 from the cardia. Additional targeted biopsies were obtained of visible abnormalities and lesions in the stomach, if present.

IM was graded according to the visual analog scale of the updated Sydney system (0: absent; 1: mild; 2: moderate; 3: marked). Mucosal atrophy score was evaluated on a four-level scale [no atrophy (0\%) score $=0$; mild atrophy (1-30\%) score $=1$; moderate atrophy (31-60\%) score $=2$; and severe atrophy $(>60 \%)$ score $=3$ ] (3). In addition, the Vienna system was used to classify dysplasia as lowor high-grade neoplasia (13). For histopathologic examination, preparations were stained with hematoxylin-eosin and Alcian blue-periodic acid Schiff ( $\mathrm{pH}$ 2.5). Giemsa staining was performed to identify H. pylori infection. The biopsy samples obtained during the first endoscopy were re-evaluated by the same pathologist (Y.S.G.), independent of any subsequent biopsy. Because different lesion grades often coexist in pathologic specimens obtained from the same patient, the highest grade lesion observed in any biopsy specimen was used to grade the disease in each patient. IM was subclassified, based on the morphologic characteristics, as "complete" (presence of mature brush border absorptive cells, sialomucin-secreting goblet cells and, occasionally, Paneth cells) or "incomplete" (few absorptive cells, secretion of sulfomucin by intermediate cells, secretion of sialomucin and/or sulfomucin by goblet cells, and marked glandular distortion and branching in the metaplastic glands) (14).

The Operative Link on Gastritis Assessment (OLGA) staging system was used to determine the disease status according to the antrum and corpus scores. The disease was graded on a scale ranging from stage 0 (none) to stage 4 (severe) (15). For the Operative Link on Gastric Intestinal Metaplasia (OLGIM) system, IM was evaluated instead of atrophy, and the severity and distribution of IM were classified on a scale ranging from stage 0 (none) to stage 4 (severe) (16). Patients with stage 3-4 disease on OLGA and OLGIM were considered at high risk of GC.

This study was approved by the Kocaeli University Faculty of Medicine Local Ethics Committee (approval number: 4, date: 2011). Informed consent was obtained.

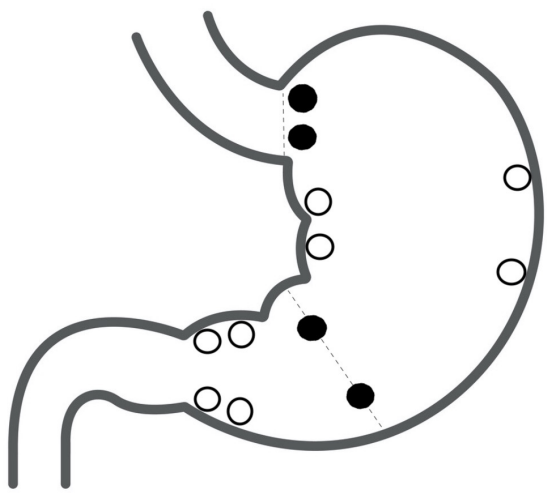

Figure 1: Biopsy sites (adapted from reference 12) 


\section{Statistical Analysis}

Statistical analysis was performed using SPSS software (version 20.0; SPSS Inc., Chicago, IL, USA). We used the Shapiro-Wilk test to determine if numerical variables followed a normal distribution. The Wilcoxon test was used to evaluate the dependent numerical variables that were not normally distributed. The relationship between categorical variables was determined using the chisquare analysis. Relationships between numerical variables were tested using the Spearman's rank correlation coefficient. Numerical variables are expressed as mean and standard deviation, and categorical variables are expressed as numbers and percentages. A $p$-value $<0.05$ indicated statistical significance.

\section{RESULTS}

\section{Baseline Characteristics at Initial Endoscopy}

The study included 117 patients with IM restricted to the antrum, with or without atrophy on initial endoscopy, which was performed at the Department of Gastroenterology, Kocaeli University Faculty of Medicine. The median [interquartile range (IQR)] age of patients at the time of surveillance endoscopy was 59 years (49-67 years), and $57.3 \%$ of patients were women. At baseline, 95 patients $(81 \%)$ had a histologic diagnosis of atrophy, $79(67.5 \%)$ had incomplete IM, 38 (32.5\%) had complete IM, and 19 (16.2\%) had low-grade dysplasia (LGD). On initial endoscopy, $41.0 \%$ of patients had $H$. pylori infection, of which $44.8 \%$ had undergone $H$ pylori eradication therapy after initial endoscopy. Overall, 51.7\% of patients were smokers, $36.8 \%$ were using NSAIDs, and $69.2 \%$ were on PPIs at initial endoscopy. One-quarter (25.0\%) of patients had a family history of GC. Nevertheless, no relationship was noted between history of GC and IM type ( $p=0.301)$ or IM grade $(p=0.929)$ (Table 1).

\section{Surveillance Endoscopy}

Surveillance endoscopy was performed at a median (IQR) of 7.2 years (5.9-8.7) after the initial diagnosis of IM. On initial endoscopy, 22 patients had antrum-restricted IM with atrophy, and 95 patients had no atrophy. On surveillance endoscopy, the rates of dysplasia were similar between patients with and without atrophy $(p=0.339)$. IM was absent in 22 patients (18.8\%) on surveillance endoscopy. Among the 95 patients with IM, 58.9\% of patients had IM present in only the antrum or incisura angularis, $4.2 \%$ in the corpus, and $36.8 \%$ had in both regions.

Surveillance endoscopy revealed high-grade dysplasia (HGD) in four patients (3.4\%) with previous indefinite dysplasia and gastric adenocarcinoma in two patients (1.7\%) with previous LGD. The annual incidence of $\mathrm{GC}$ in patients with antrum-restricted IM was $0.17 \%$. In the first patient with gastric adenocarcinoma, diffuse gastric carcinoma was detected in the incisura angularis 4.6 years after the initial endoscopy, and surgery was performed. In the second patient, an intestinal-type early gastric carcinoma was detected 4.7 years after onset, and endoscopic submucosal dissection was performed.

\section{Progression and Regression of Premalignant Lesions}

On surveillance endoscopy, $11.1 \%$ of patients exhibited progression of IM type, $27.4 \%$ had progression of IM grade, $25.6 \%$ had progression of atrophy, and $33.3 \%$ had progression of dysplasia compared with the results of the initial endoscopy. Compared with the findings on initial endoscopy, IM type regressed in $38.5 \%$ of patients, and IM grade regressed in $29.9 \%$. Notably,

\begin{tabular}{|c|c|}
\hline Age, mean $( \pm S D), y$ & $58.26 \pm 11.2$ \\
\hline \multicolumn{2}{|l|}{ Sex } \\
\hline Male & $50(42.7)^{\star}$ \\
\hline Female & $67(57.3)^{\star}$ \\
\hline \multicolumn{2}{|l|}{ IM type } \\
\hline Complete & $38(32.5)^{\star}$ \\
\hline Incomplete & $79(67.5)^{\star}$ \\
\hline \multicolumn{2}{|l|}{ IM grade } \\
\hline Mild & $48.0(41.0)^{*}$ \\
\hline Moderate & $48.0(41.0)^{\star}$ \\
\hline Marked & $21(18.0)^{\star}$ \\
\hline \multicolumn{2}{|l|}{ Atrophy } \\
\hline None & $22(18.8)^{\star}$ \\
\hline Mild & $65(55.5)^{\star}$ \\
\hline Moderate & $26(22.2)^{\star}$ \\
\hline Marked & $4(3.4)^{*}$ \\
\hline \multicolumn{2}{|l|}{ Dysplasia } \\
\hline None & $31(26.5)^{\star}$ \\
\hline Undefined & $67(57.3)^{\star}$ \\
\hline Low grade & $19(16.2)^{\star}$ \\
\hline \multicolumn{2}{|l|}{ Helicobacter pylori } \\
\hline Negative & $69(59)^{\star}$ \\
\hline Positive & $48(41)^{\star}$ \\
\hline \multicolumn{2}{|c|}{ Family history of gastric cancer } \\
\hline Yes & $29(25.0)^{\star}$ \\
\hline No & $87(75.0)^{\star}$ \\
\hline \multicolumn{2}{|l|}{ NSAIDs } \\
\hline Yes & $43(36.8)^{\star}$ \\
\hline No & $74(63.3)^{\star}$ \\
\hline \multicolumn{2}{|c|}{ Proton pump inhibitors } \\
\hline Yes & $81(69.2)^{*}$ \\
\hline No & $36(30.8)^{\star}$ \\
\hline \multicolumn{2}{|l|}{ Smoking } \\
\hline Yes & $60(51.7)^{\star}$ \\
\hline No & $56(48.3)^{\star}$ \\
\hline \multicolumn{2}{|l|}{ Alcohol consumption } \\
\hline Yes & $13(11.2)^{\star}$ \\
\hline No & $103(88.8)^{\star}$ \\
\hline
\end{tabular}


IM type and grade were stable in $50.4 \%$ and $42.7 \%$ of patients, respectively. Similarly, atrophy regressed in $35.0 \%$ of patients, and atrophy was stable in $39.3 \%$ based on surveillance endoscopy; dysplasia regressed in $23.1 \%$ of patients, and dysplasia was stable in $43.6 \%$ (Table 2). Among patients with incomplete metaplasia on initial endoscopy, $54.0 \%$ had incomplete IM on surveillance endoscopy, 25.3\% had complete IM, and 20.3\% did not have IM. Among the patients with complete IM on initial endoscopy, 34.2\% progressed to incomplete metaplasia, and $23.7 \%$ did not have IM.

On surveillance endoscopies, a positive correlation was observed between the progression of the IM grade and the progression of the IM type ( $r=0.59, p=0.001)$, atrophy $(r=0.52, p=0.001)$, and dysplasia $(r=0.55, p=0.001)$. Likewise, as IM type progression, both atrophy $(r=0.22, p<0.05)$ and dysplasia progressed $(r=0.41$, $p=0.001$ ). Moreover, as atrophy progressed, dysplasia progressed too $(r=0.53, p=0.001)$.

\section{Progression to Dysplasia in Premalignant Lesions}

A statistically significant difference was observed between IM grade on the initial biopsies and the distribution of dysplasia in subsequent biopsies $(p=0.002)$ and between atrophy status on the initial biopsies and the distribution of dysplasia in subsequent biopsies $(p=0.019)$. Fourteen patients $(43.8 \%)$ who had LGD on surveillance endoscopy, as well as all four patients with HGD (100\%) and two patients with GC (100\%), had moderate IM on initial endoscopy. However, 23 patients (56.1\%) without dysplasia and 17 (44.7\%) with indefinite dysplasia had mild IM on initial endoscopy ( $p=0.002$ ). Likewise, 13 patients $(40.6 \%$ ) with $L G D$ and 2 patients $(100 \%)$ with GC had moderate atrophy scores on the initial biopsies. Moreover, 20 patients (48.8\%) without dysplasia and 28 patients $(73.7 \%)$ with indefinite dysplasia had mild atrophy scores on the initial biopsies $(p=0.019)$. No significant relationship was noted between IM type on the initial biopsies and the distribution of dysplasia on subsequent biopsies ( $p=0.070$ ) (Table 3). However, 24 of 32 patients (75\%) who had LGD on surveillance endoscopy had incomplete IM on initial endoscopy, and in all 4 patients (100\%) with HGD and both patients (100\%) with GC on surveillance endoscopy, incomplete IM was noted on initial endoscopy.

\section{Risk Factors}

The rate of $H$. pylori infection was significantly higher on surveillance endoscopy compared with initial endoscopy $(p=0.001)$. Notably, including the 23 patients who received eradication therapy before initial endoscopy, 53 patients (76.8\%)

\section{Table 2. Progression and regression of premalignant gastric lesions on surveillance endoscopy}

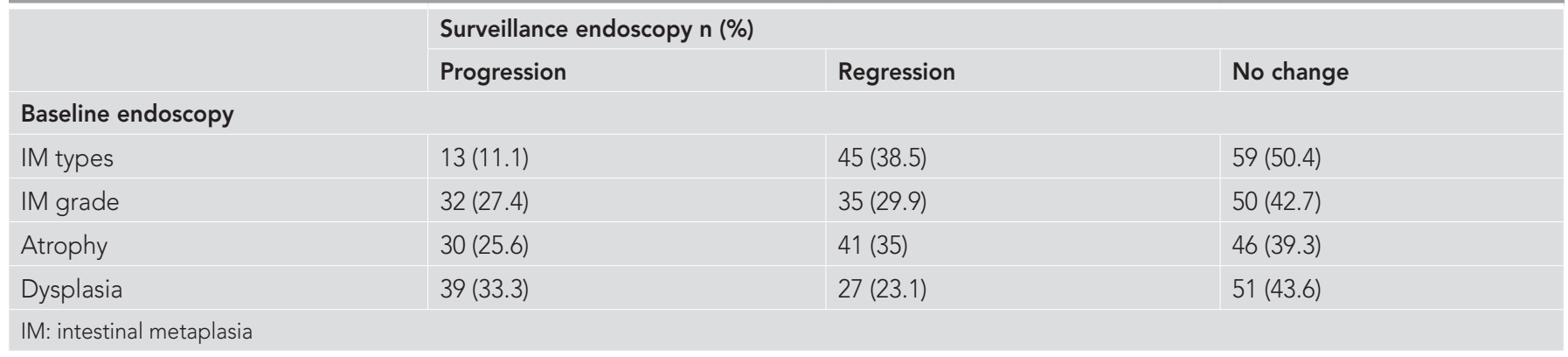

Table 3. Comparison of patients at low and high risk for gastric cancer on surveillance endoscopy according to histologic characteristics on initial endoscopy

\begin{tabular}{|c|c|c|c|c|c|c|}
\hline \multicolumn{7}{|c|}{ Dysplasia on surveillance endoscopy, $n$ (\%) } \\
\hline Baseline endoscopy & $G C(n=2)$ & HGD $(n=4)$ & LGD $(n=32)$ & Indefinite $(n=38)$ & None $(n=41)$ & $p$ \\
\hline \multicolumn{7}{|l|}{ IM grade } \\
\hline Moderate & $2(100)$ & $4(100)$ & $14(43.8)$ & $14(36.8)$ & $14(34.1)$ & $0.002^{*}$ \\
\hline Mild & 0 & 0 & $8(25)$ & $17(44.7)$ & $23(56.1)$ & - \\
\hline Complete & 0 & 0 & $8(25)$ & $13(34.2)$ & $17(41.5)$ & - \\
\hline \multicolumn{7}{|l|}{ Atrophy } \\
\hline Marked & 0 & 0 & $1(3.1)$ & 0 & $3(7.3)$ & - \\
\hline Moderate & $2(100)$ & 0 & $13(40.6)$ & $4(10.5)$ & $7(17.1)$ & - \\
\hline
\end{tabular}


without $H$. pylori infection had positive $H$. pylori results on surveillance endoscopy.

Nevertheless, no correlation was noted between the progression and regression status of premalignant lesions and possible risk factors, such as age, sex, smoking history, alcohol use, PPI and NSAID use, and $H$. pylori infection (all $p>0.05$ ).

\section{OLGA and OLGIM Stage}

On surveillance endoscopy, $32.8 \%$ of patients had OLGA stage 1 disease, $24.1 \%$ had stage $2,10.3 \%$ had stage 3 , and $1.7 \%$ had stage 4. No significant relationship was observed between IM grade $(p=0.064)$, IM type $(p=0.593)$, atrophy $(p=0.222)$, or dysplasia distribution ( $p=0.138$ ) on initial endoscopy and the OLGA stages on surveillance endoscopy. However, on surveillance endoscopy, OLGA stage 3-4 disease was noted in most patients who had progression of $I M$ grade $(p=0.0001)$, IM type $(p=0.008)$, and dysplasia $(p=0.0001)$.

On surveillance endoscopy, 19 patients (16.2\%) had OLGIM stage 1, 41 (35\%) had stage 2, 22 (18.8\%) had stage 3, and $14(12 \%)$ had stage 4. Furthermore, with the increase in IM grade, IM type, atrophy, and dysplasia on initial endoscopy, an increase in OLGIM stage was observed on surveillance endoscopy. Most patients with OLGIM stage 2-4 disease had incomplete IM, and LGD, or indefinite dysplasia on initial endoscopy, whereas most OLGIM stage 1 patients had complete IM $(p=0.044)$ and no dysplasia $(p=0.009)$ (Table 4).
Regarding the OLGIM stage of premalignant lesions on surveillance endoscopy, moderate atrophy was noted in most patients with OLGIM stages $3(59.1 \%)$ and 4 (50.0\%); stage 2 was seen in most patients (56.1\%) with mild atrophy, and atrophy was not observed in most patients with stage 1 (63.2\%) ( $p=0.001)$. Incomplete IM was observed in most patients with OLGIM stages $2(51.2 \%), 3(86.4 \%)$, and $4(85.7 \%)$, and complete IM was observed in most patients with stage 1 (73.7\%) ( $p=0.001)$. Regarding dysplasia, LGD was noted in most patients with stages $3(50.0 \%)$ and $4(57.1 \%)$, and nearly half (48.8\%) of patients with indeterminate dysplasia had stage 2 . Typically, dysplasia was not observed in patients with stage $1(p=0.001)$.

Furthermore, most patients whose atrophy progressed on surveillance endoscopy had OLGIM stages $3(45.5 \%)$ and 4 (42.9\%) $(p=0.001)$, and those whose dysplasia progressed were OLGIM stages $3(50.0 \%)$ and $4(64.3 \%)(p=0.001)$.

\section{DISCUSSION}

In this study, patients with antrum-restricted IM on untargeted biopsies obtained during initial endoscopy were re-evaluated after a median of 7.2 years. Based on our results, patients with antrum-restricted IM are at risk of neoplastic lesions and require endoscopic surveillance. In addition, it has been observed that premalignant lesions can both progress and regress during clinical surveillance.

\begin{tabular}{|c|c|c|c|c|c|c|}
\hline \multicolumn{7}{|c|}{ OLGIM on surveillance endoscopy, $n(\%)$} \\
\hline Baseline endoscopy & Stage 4 & Stage 3 & Stage 2 & Stage 1 & None & $\mathrm{p}$ \\
\hline \multicolumn{7}{|l|}{ IM grade } \\
\hline Marked & $6(42.9)$ & $7(31.8)$ & $5(12.2)$ & $1(5.3)$ & $2(9.5)$ & - \\
\hline Moderate & $6(42.9)$ & $9(40.9)$ & $22(53.7)$ & $3(15.8)$ & $8(38.1)$ & $0.001^{*}$ \\
\hline Mild & $2(14.3)$ & $6(27.3)$ & $14(34.1)$ & $15(78.9)$ & $11(52.4)$ & - \\
\hline \multicolumn{7}{|l|}{ IM type } \\
\hline Incomplete & $12(85.7)$ & $19(86.4)$ & $26(63.4)$ & $9(47.4)$ & $13(61.9)$ & 0.044 \\
\hline Complete & $2(14.3)$ & $3(13.6)$ & $15(36.6)$ & $10(52.6)$ & $8(38.1)$ & - \\
\hline \multicolumn{7}{|l|}{ Atrophy } \\
\hline Marked & $0(0.0)$ & $1(4.5)$ & $0(0.0)$ & $1(5.3)$ & $2(9.5)$ & - \\
\hline Moderate & $5(35.7)$ & $5(22.7)$ & $11(26.8)$ & $0(0.0)$ & $5(23.8)$ & 0.043 \\
\hline Mild & $8(57.1)$ & $11(50.0)$ & $25(61.0)$ & $13(68.4)$ & $8(38.1)$ & - \\
\hline None & $1(7.1)$ & $5(22.7)$ & $5(12.2)$ & $5(26.3)$ & $6(28.6)$ & - \\
\hline \multicolumn{7}{|l|}{ Dysplasia } \\
\hline High-grade & $0(0.0)$ & $0(0.0)$ & $0(0.0)$ & $0(0.0)$ & $0(0.0)$ & - \\
\hline Low grade & $4(28.6)$ & $6(27.3)$ & $5(12.2)$ & $1(5.3)$ & $3(14.3)$ & $0.009 *$ \\
\hline Indefinite & $9(64.3)$ & $11(50.0)$ & $29(70.7)$ & $6(31.6)$ & $12(57.1)$ & - \\
\hline None & $1(7.1)$ & $5(22.7)$ & $7(17.1)$ & $12(63.2)$ & $6(28.6)$ & - \\
\hline
\end{tabular}


Delayed diagnosis of GC is associated with a high mortality rate. Therefore, it is imperative to screen for premalignant lesions in high-risk groups (17). Moreover, an uneven IM distribution might cause sampling errors, making the detection of premalignant gastric lesions challenging. Therefore, the best approach is to use diagnostic endoscopy with a gastric mapping protocol. The updated Sydney system is a widely applied biopsy protocol (7). However, studies have reported that this protocol does not fully reflect the actual state of IM $(18,19)$. Therefore, in our study, we used a biopsy protocol to obtain specimens from all stomach regions to optimally assess the severity and distribution of premalignant gastric lesions (12). Most premalignant gastric lesions were observed in the antrum, followed by throughout the stomach and the corpus. In Western countries and other populations, $50 \%$ of precancerous gastric lesions were noted in the antrum, $17.7 \%$ in the corpus, and $15 \%$ in both regions $(20,21)$.

Notably, the prevalence of $\mathrm{GC}$ varies significantly among different geographic regions $(22,23)$. Guidelines recommend surveillance endoscopy for patients with extensive IM and atrophy, and also for patients with gastric IM who are at high risk for GC owing to their ethnicity or family history $(5,6,10,11)$. European guidelines recommend an interval of 3 years and more more intensive surveillance for those with extensive gastric IM and atrophy, whereas the guidelines of the American Gastroenterological Association recommend a 3- to 5-year surveillance period $(5,6)$. Nonetheless, these guidelines do not recommend follow-up for patients with antrum-restricted gastric IM and atrophy. However, in our study, $27.4 \%$ of patients with antrum-restricted IM on initial endoscopy had progression of the IM grade, whereas $25.6 \%$ had progression of atrophy and $33.3 \%$ had progression of dysplasia on surveillance endoscopy. In addition, HGD and GC were detected in four and two patients, respectively. For the two patients with GC, the mean time between initial endoscopy and GC diagnosis was 4.65 years. In our series, the annual incidence of $\mathrm{GC}$ was $0.17 \%$ among patients with antrum-restricted IM. In one study, the 10 -year incidence of GC was $0.8 \%$ among patients with atrophic gastritis, while that of patients with IM was $1.8 \%$ (8). Therefore, it might not be appropriate to use the same follow-up period for patients with atrophic gastritis and IM. However, per another study, no patients with atrophic gastritis or complete IM on their initial biopsies developed HGD or GC during the 3-year followup period, with only less than $10 \%$ of cases progressing to LGD (24). Nevertheless, the annual endoscopic follow-up might not be appropriate for all patients with IM because some will not develop GC. Therefore, a better approach would be to devise a patientindividualized follow-up strategy.

Although the US guidelines on surveillance endoscopy consider patients with incomplete IM as high risk, the European guidelines and the study of Dinis-Ribeiro et al. (11) do not consider IM type $(5,6,12)$. Gonzalez et al. $(25)$ revealed that the risk of $G C$ is three times higher in patients with incomplete IM than those with complete IM. In another study from Spain, 16 of 21 patients with adenocarcinoma had incomplete IM at a mean of 12.8 years after the initial diagnosis, and 1 patient had complete $\mathrm{IM}$; the risk of $G C$ was highest among patients with incomplete IM, and a family history of GC (26). In our study, all four patients with HGD and two with GC, diagnosed on surveillance endoscopy, had incomplete metaplasia on the initial endoscopy. Dinis-Ribeiro et al. (11) suggested using IM grade instead of IM subtype. However, we determined a positive correlation between IM type and grade in our study. Notably, with the progression of IM type, the IM grade and dysplasia also progressed. Therefore, contrary to recommendations, patients with complete IM in the antral mucosa, a history of smoking, a family history of $\mathrm{GC}$, or incomplete IM restricted to the antrum would require endoscopic surveillance (27).

While some precancerous gastric lesions show progression, others may remain stable, or exhibit true regression or show false regression according to the characteristics of the biopsy sampling site or interpretation of histologic grades $(21,28)$. Our data indicated that premalignant lesions might exhibit both progression and regression on clinical surveillance. In our series, based on the findings of the initial endoscopies, $38.5 \%$ of patients had IM type regression, and $29.9 \%$ had IM grade regression. Nevertheless, IM type remained stable in $50.4 \%$ of patients, and IM grade remained stable in 42.7 . Similarly, $35 \%$ of patients had atrophy regression, and $39.3 \%$ had persistent atrophy, whereas $23.1 \%$ of patients had dysplasia regression, and $43.6 \%$ had persistent dysplasia. Studies have revealed that the location, severity, and extent of precancerous lesions, particularly IM, reflect the likelihood of progression to GC (29). Contrary to some studies stating that IM does not regress, recent studies have demonstrated that IM can be reversible $(30,31)$. When Akbari et al. (28) used a random-effects model to review 20 studies on patients with IM, they reported that the IM regressed in $31.8 \%$, whereas it remained stable in $43.4 \%$. When the results of 10 studies were combined, $32.2 \%$ of patients had atrophy regression, and $38.8 \%$ had persistent atrophy (28). In addition, the characteristics of LGD and regenerative changes exhibit a large overlap, which could complicate the diagnosis of LGD $(32,33)$. Nevertheless, a study that followed up patients with dysplasia for more than 2 years noted that among patients with LGD, $21 \%$ had progression and $36 \%$ had spontaneous regression, and in those with moderate-grade dysplasia, 33\% had progression, and $27 \%$ had spontaneous regression. In addition, $43 \%$ of cases with severe dysplasia remained stable, $47 \%$ progressed to GC, and $0 \%$ regressed (34). Strikingly, den Hoed et al. (19) revealed that $67 \%$ of cases of $L G D$ regressed to $I M$, and the remaining third had regression to atrophic gastritis and even normal mucosa on surveillance endoscopy.

In our study, the severity and extent of premalignant lesions detected on initial endoscopy were not associated with OLGA stage but were associated with OLGIM stage. On initial endoscopy, we observed that, as the severity of IM grade, IM type, atrophy, and dysplasia increased, so did the OLGIM stage on surveillance endoscopy. In addition, patients whose premalignant lesions exhibited progression on surveillance endoscopy had 
2. Correa P. A human model of gastric carcinogenesis. Cancer Res 1988; 48: 3554-60.

3. Dixon MF, Genta RM, Yardley JH, Correa P. Classification and grading of gastritis. The updated Sydney System. International Workshop on the Histopathology of Gastritis, Houston 1994. Am J Surg Pathol 1996; 20: 1161-81.

4. Zhang X, Li M, Chen S, Hu J, Guo Q, Liu R, et al. Endoscopic screening in Asian countries is associated with reduced gastric cancer mortality: a meta-analysis and systematic review. Gastroenterology 2018; 155: 34754.

5. Gupta S, Li D, El Serag HB, Davitkov P, Altayar O, Sultan S, et al. AGA Clinical Practice Guidelines on management of gastric intestinal metaplasia. Gastroenterology 2020; 158: 693-702.

6. Pimentel-Nunes P, Libânio D, Marcos-Pinto R, Areia M, Leja M, Esposito G. Management of epithelial precancerous conditions and lesions in the stomach (MAPS II): European Society of Gastrointestinal Endoscopy (ESGE), European Helicobacter and Microbiota Study Group (EHMSG), European Society of Pathology (ESP), and Sociedade Portuguesa de Endoscopia Digestiva (SPED) guideline update 2019. Endoscopy 2019; 51: 365-88.

7. Vance RB Jr, Kubiliun N, Dunbar KB. How do we manage gastric intestinal metaplasia? a survey of clinical practice trends for gastrointestinal endoscopists in the United States. Dig Dis Sci 2016; 61: 1870-8.

8. De Vries A, Van Gfieken N, Looman CW, Casparie MK, De Vries E, Meijer $\mathrm{GA}$, et al. Gastric cancer risk in patients with premalignant gastric lesions: a nationwide cohort study in the Netherlands. Gastroenterology 2008; 134: 945-52.

9. Song H, Ekheden IG, Zheng Z, Ericsson J, Nyrén O, Ye W. Incidence of gastric cancer among patients with gastric precancerous lesions: observational cohort study in a low risk Western population. BMJ 2015; 351: h3867.

10. Evans JA, Chandrasekhara V, Chathadi KV, Decker GA, Early DS, Fisher DA, et al. The role of endoscopy in the management of premalignant and malignant conditions of the stomach. Gastrointest Endosc 2015; 82: $1-8$.

11. Dinis-Ribeiro $M$, Areia $M$, De Vries AC, Marcos-Pinto $R$, MonteiroSoares $\mathrm{M}, \mathrm{O}^{\prime}$ Connor A. Management of precancerous conditions and lesions in the stomach (MAPS): guideline from the European Society of Gastrointestinal Endoscopy (ESGE), European Helicobacter Study Group (EHSG), European Society of Pathology (ESP), and the Sociedade Portuguesa de Endoscopia Digestiva (SPED). Endoscopy 2012; 44: 74-94.

12. De Vries AC, Haringsma J, De Vries RA, ter Borg F, Nagtzaam NM, Steyerberg EW, et al. The use of clinical, histologic, and serologic parameters to predict the intragastric extent of intestinal metaplasia: a recommendation for routine practice. Gastrointest Endosc 2009; 70: 1825

13. Schlemper R, Riddell R, Kato Y,Borchard F, Cooper H, Dawsey S. The Vienna classification of gastrointestinal epithelial neoplasia. Gut 2000; 47: 251-5.

14. Jencks DS, Adam JD, Borum ML, Koh JM, Stephen S, Doman DB: Overview of current concepts in gastric intestinal metaplasia and gastric cancer. Gastroenterol Hepatol 2018; 14: 92-101.

15. Rugge M, MeggioA, Pennelli G, Piscioli F, Giacomelli L, De Pretis G, et al. Gastritis staging in clinical practice: the OLGA staging system. Gut 2007; 56: 631-6.

16. Rugge M, Fassan M, Pizzi M, Farinati F, Sturniolo GC, Plebani M, et al. Operative link for gastritis assessment vs operative link on intestinal metaplasia assessment. World J Gastroenterol 2011; 17: 4596-601.

17. Takahashi T, Saikawa Y, Kitagawa Y. Gastric cancer: current status of diagnosis and treatment. Cancers 2013; 5: 48-63.

18. El-Zimaity HM, Graham DY. Evaluation of gastric mucosal biopsy site and number for identification of Helicobacter pylori or intestinal metaplasia: role of the Sydney System. Hum Pathol 1999; 8177: 90303-9.

19. den Hoed CM, Holster IL, Capelle LG, De Vries AC, den Hartog B, Ter Borg F, et al. Follow-up of Premalignant Lesions in Patients at Risk for Progression to Gastric Cancer. Endoscopy 2013; 45: 249-56. 
20. Chapellea N, Péron M, Mosnier JF, Quénéhervé L, Coron E, Bourget $A$, et al. Prevalence, characteristics and endoscopic management of gastric premalignant lesions in France. Digest Dis 2019; 1-7.

21. Mera RM, Bravo LE, Camargo MC, Bravo JC, Delgado AG, RomeroGallo J, et al. Dynamics of Helicobacter pylori infection as a determinant of progression of gastric precancerous lesions: 16-year follow-up of an eradication trial. Gut 2018; 67: 1239-46.

22. Sitarz R, Skierucha M, Mielko J, Offerhaus GJA, Maciejewski R, Polkowski WP. Gastric cancer: epidemiology, prevention, classification, and treatment. Cancer Manag Res 2018; 10: 239-48.

23. Rubio CA, Hirota T, Itabashi M, Mandai K, Yanagisawa A, Kitagawa T, et al. Extended Intestinal Metaplasia. A Survey of 1392 gastrectomies from dwellers of the Pacific Basin. Anticancer Res 2004; 24: 3185-92.

24. Dinis-Ribeiro M, Lopes $C$, da Costa-Pereira A, Guilherme M, Barbosa J, Lomba-Viana $\mathrm{H}$, et al. A follow up model for patients with atrophic chronic gastritis and intestinal metaplasia. J Clin Pathol 2004; 57: 177-82.

25. Gonzalez CA, Sanz-Anquela JM, Companioni O, Bonet C, Berdasco M, López Consuelo. Incomplete type of intestinal metaplasia has the highest risk to progress to gastric cancer: results of the Spanish follow-up multicenter study. J Gastroenterol Hepatol 2016; 31: 953-58.

26. Gonzalez CA, Pardo ML, Liso JM, Alonso P, Bonet C, Garcia RM, et al. Gastric cancer occurrence in preneoplastic lesions: a long-term follow-up in a high-risk area in Spain. Int J Cancer 2010; 127: 2654-60.

27. Zullo A, Hassan C, Repici A, Annibale B. Intestinal metaplasia surveillance: Searching for the road- map. World J Gastroenterol 2013; 19: 1523-6.
28. Akbari M, Tabrizi R, Kardeh S, Lankarani KB. Gastric cancer in patients with gastric atrophy and intestinal metaplasia: A systematic review and meta-analysis. PLoS One 2019; 14: e0219865.

29. Sakitani K, Hirata Y, Watabe H, Yamada A, Sugimoto T, Yamaji Y, et al. Gastric cancer risk according to the distribution of intestinal metaplasia and neutrophil infiltration. J Gastroenterol Hepatol 2011; 26: 1570-5.

30. Fuccio L, Zagari RM, Minardi ME, Bazzoli F. Systematic review: Helicobacter pylori eradication for the prevention of gastric cancer. Alimentary Pharmacol Ther 2007; 25: 133-41.

31. Kiriyama Y, Tahara T, Shibata T, Okubo M, Nakagawa M, Okabe A, et al. Gastric-and-intestinal mixed intestinal metaplasia is irreversible point with eradication of Helicobacter pylori. O J Pathol 2016; 6: 93.

32. Rugge M, Correa P, Dixon MF, Hattori T, Leandro G, Lewin K, et al. Gastric dysplasia: the Padova international classification. Am J Surg Pathol 2000; 24: 167-76.

33. Lauwers GY, Riddell RH. Gastric Epithelial Dysplasia. Gut 1999; 45: 78490.

34. Rugge M, Farinati F, Baffa R, Sonego F, Di Mario F, Leandro G, et al. Gastric epithelial dysplasia in the natural history of gastric cancer: a multicenter prospective follow-up study. Interdisciplinary Group on Gastric Epithelial Dysplasia. Gastroenterology 1994; 107: 1288-96.

35. Isajevs S, Liepniece-Karele I, Janciauskas D, Moisejevs G, Putnins V, Funka K, et al. Gastritis staging: interobserver agreement by applying OLGA and OLGIM systems. Virchows Arch 2014; 464: 403-7. 